\title{
MAGNETO-OPTIC INVESTIGATION OF MAGNETIC FLUX PENETRATION ON A NANOSECOND TIMESCALE
}

\author{
B. Biehler, U. Bolz, B.-U. Runge, and P. Leiderer \\ Department of Physics, Universitätsstr. 10, 78457 Konstanz, Germany \\ Bjoern.biehler@uni-konstanz.de
}

\begin{abstract}
We have studied the dynamics of iron-garnet films using a pump-probe technique and were able to demonstrate that the response time of such a film is sufficiently short to investigate sub-nanosecond phenomena. Furthermore, we studied the penetration velocity of the dendritic instability in thin YBCO films. We found two regimes: An early stage with a penetration velocity of up to $170 \mathrm{~km} / \mathrm{s}$ and a distinct later stage were the velocity is lower $(\approx 18 \mathrm{~km} / \mathrm{s})$.
\end{abstract}

Keywords: $\quad \mathrm{YBCO}$, time resolution, flux penetration

\section{Introduction}

The first spatially resolved observation of flux jumps was reported in 1967 by Wertheimer $e t$ al. [1], who studied superconducting Niobium discs with a thickness of several tenths of a millimeter by means of magneto-optics. These authors found finger-like flux fronts with a typical width of $1 \mathrm{~mm}$ which propagated with a typical speed between 5 and $100 \mathrm{~m} / \mathrm{s}$. Later on studies of thin superconducting Niobium and YBCO films showed a similar flux jump phenomenon. It most often results in a dendritic flux pattern, independent of how the avalanche developed, i.e. spontaneously [2-4] or triggered by local heating of the sample with a short laser pulse [5,6].

That dendritic flux avalanches are a rather general phenomenon in superconductors was corroborated recently by similar observations in a couple of materials $\left(\mathrm{MgB}_{2}[7]\right.$ or $\left.\mathrm{YNi}_{2} \mathrm{~B}_{2} \mathrm{C}\right)$. 


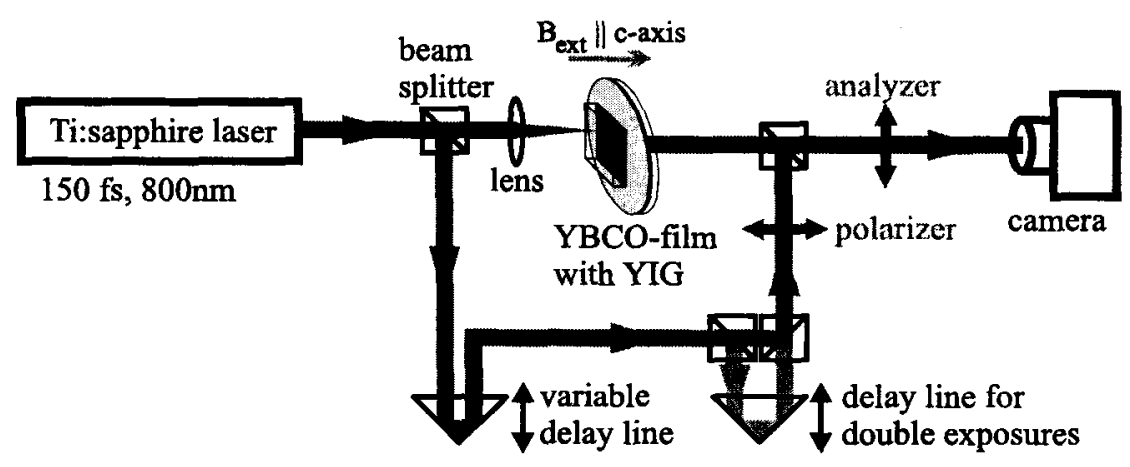

Figure 1. Experimental setup. For details see text.

\section{Experimental setup}

The requirement for the investigation of dendrite dynamics is a temporal resolution in the sub-nanosecond regime. This is due to the high velocities occurring during the development of flux avalanches. We have used a magneto-optic technique, with a Bi-doped Yttrium Iron Garnet (YIG) film on Gadolinium Gallium Garnet as the sensor layer [9], which was mounted on top of the superconductor [10].

The spatial resolution was on the order of a few $\mu \mathrm{m}$. The high temporal resolution was achieved by a pump-probe technique in combination with a Ti:sapphire pulse laser $(t=150 \mathrm{fs})$ as an illumination source. The experimental setup is sketched in Fig. 1: The laser pulse is split into two. One part is used to trigger a flux avalanche, whereas the second part is fed through an optical delay line and is then used to take a magneto-optic snapshot of the flux distribution. In order to provide a measure on the instantaneous velocity double exposures were made by further splitting the imaging pulse into two, one being delayed with respect to the other by only a few nanoseconds, as it is illustrated in Fig. 3. This technique has been used to obtain the data in Fig. 4b. The samples were $10 \times 10 \mathrm{~mm}^{2}$ YBCO thin films on strontium titanate deposited by the PLD technique. The physical limit in temporal resolution with this setup is given by the response time of the YIG film, which was found to be less than $200 \mathrm{ps}$ in our case [8].

\section{Garnet dynamics}

To measure the dynamics of a garnet film a fast magnetic switch was needed to apply a magnetic field sufficiently fast. To accomplish that a $330 \mathrm{nmYBCO}$ film has been structured in circles of $0.25 \mathrm{~mm}, 0.5 \mathrm{~mm}$, and $0.75 \mathrm{~mm}$ radius. 


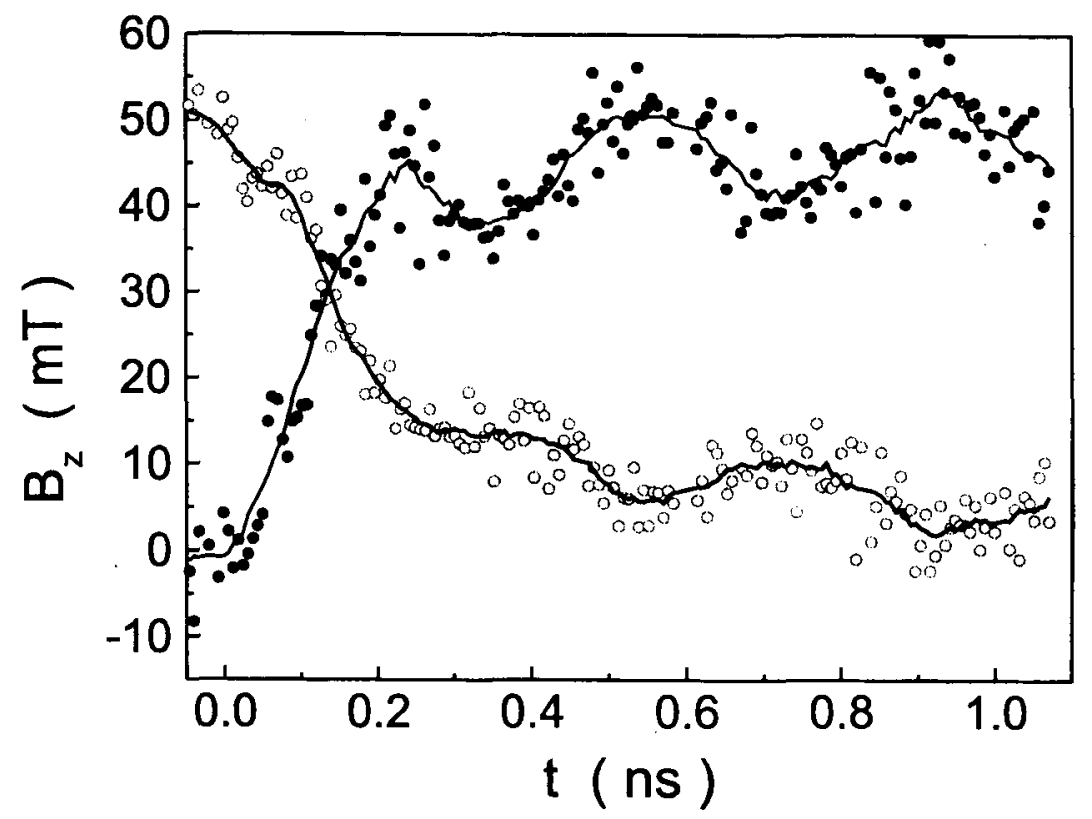

Figure 2. z-component of the magnetic field as indicated by Faraday rotation measurements. The closed symbols show the behaviour of a 39 by $39 \mu \mathrm{m}^{2}$ area from the interior of the sample, when heated in an external magnetic field. The field penetrates from the edges and the Faraday rotation increases. The open symbols show the same spot, but when the sample was field cooled and field is leaving the sample during heating. The lines are meant as a guide to the eye and represent a ten point average of the data points.

We cooled the sample below $T_{\mathrm{c}}$ and applied a magnetic field of $50 \mathrm{mT}$. Now the entire circle was heated above $T_{\mathrm{c}}$; the shielding currents decay rapidly because of the resistivity of the YBCO film. The field distribution was measured by our snapshot technique after a varying delay (closed symbols in fig. 2). Now the field was switched off and another laser pulse was applied (open symbols in fig. 2). The time it takes the current to decay can be estimated from the inductance of the circle to be on the order of $0.1 \mathrm{~ns}$.

In the first picoseconds after magnetic field penetrates into the superconductor the electron spin starts to orient itself parallel to the external field. The observed oscillation is due to a precessing motion of the spins. The response time for this particular garnet film was found to be $\approx 0.2 \mathrm{~ns}$ and is therefore short enough for the investigation of dendrite dynamics. 


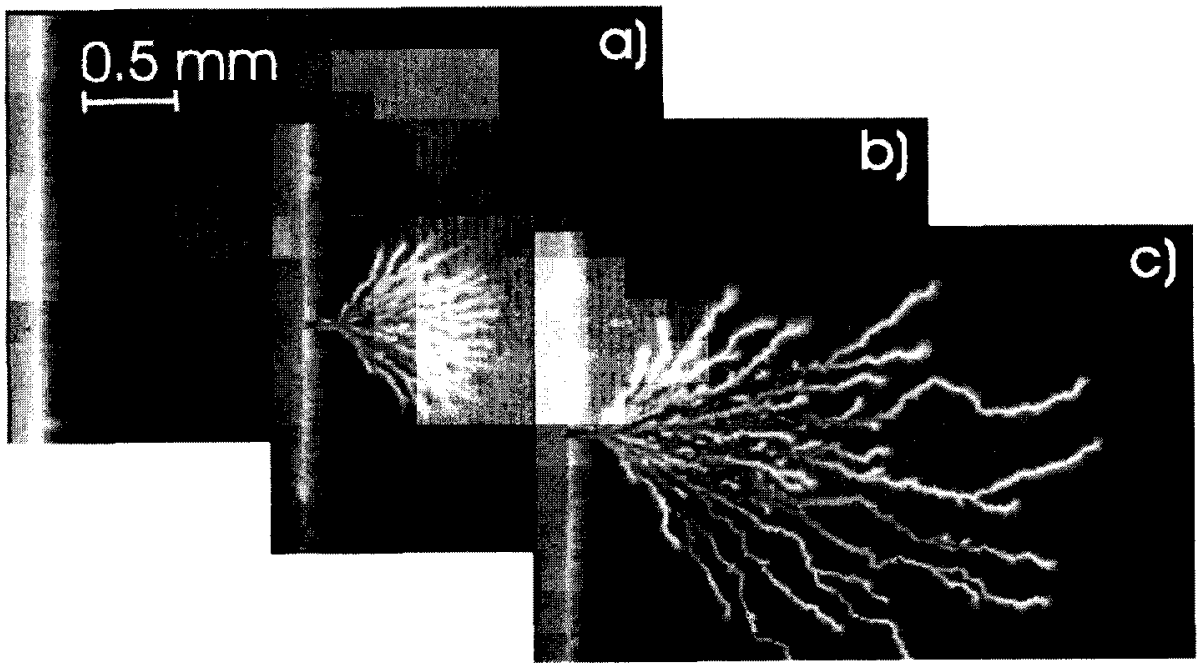

Figure 3. A typical sequence of magneto-optical images at $10 \mathrm{~K}$ in an external magnetic field of $20.6 \mathrm{mT}$. Regions of high magnetic field appear bright. Picture size is $4 \times 2.5 \mathrm{~mm}^{2}$. a) The YBCO film before the "nucleation pulse" is applied. One can clearly notice the enhancement of the field at the sample edge (left hand side of the picture). b) This is a double exposure picture. The exposures were taken $37.6 \mathrm{~ns}$ and $43.3 \mathrm{~ns}$ after nucleation of the instability. One can notice the light gray area at the dendrite tips showing the change between the two exposures. c) Here the final state of the dendrite is shown. The picture is taken $30 \mathrm{~s}$ after nucleation.

\section{Dendrite dynamics}

To study the dendrite dynamics a typical experiment was carried out in the following way: The sample was zero-field cooled below $T_{\mathrm{c}}$, and after reaching a stable temperature a magnetic field $B_{\text {ext }}$ was applied perpendicular to the film. The thin film geometry leads to an enhancement of the field at the sample edges, from where the magnetic flux penetrates partly into the superconducting film. It has to be pointed out, however, that in a thin film due to the 2-dimensional character shielding currents flow also in those areas of the film which are still in the Meissner state, i.e. the $B$-field is zero [11]. The resulting meta-stable field distribution was then disturbed locally at the sample edge by the focused laser pulse. For sufficiently strong perturbations this triggered a magnetic flux avalanche. Estimations suggest that the YBCO film was heated above $T_{\mathrm{c}}$ in the focal spot of some $50 \mu \mathrm{m}$ diameter, but an actual temperature measurement has not been carried out. However, the heating was subtle enough, not to damage the film locally, as tested by reversibility measurements.

Now, we will discuss the variation of $v$ after nucleation as the avalanche propagates towards the center of the sample. 

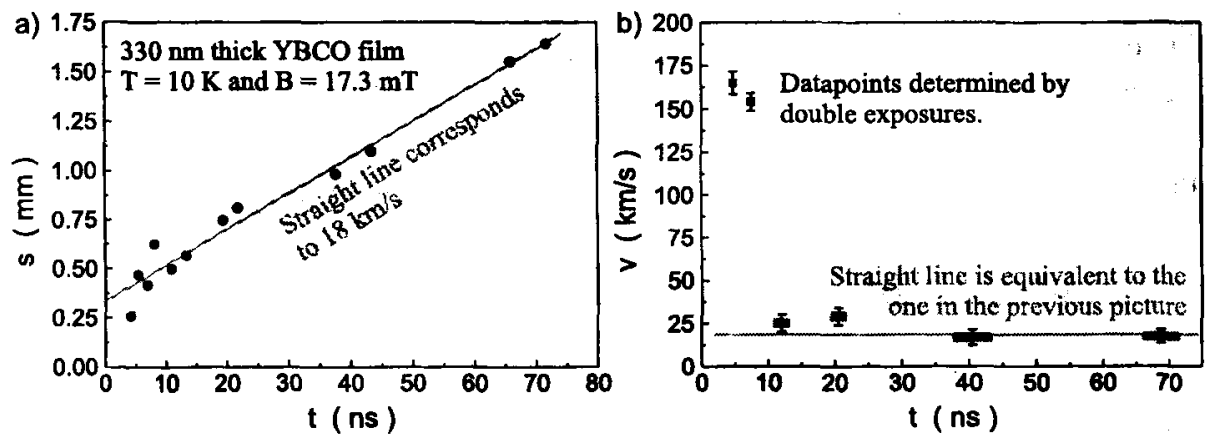

Figure 4. a) Average length $s$ of the dendrite branches. The sample was $330 \mathrm{~nm}$ thick and was held at $T=10 \mathrm{~K}$ in an external field of $B_{\text {ext }}=17.3 \mathrm{mT}$. The straight line corresponds to a velocity of $v=18 \mathrm{~km} / \mathrm{s}$. b) Data points: propagation velocity $v$ as determined by the double exposure technique (instantaneous velocity). The horizontal line is equivalent to the straight line on the left.

An example for the average length $s$ of the dendrites, determined by a sequence of single exposures taken at different delay times $t$, is shown in Fig. 4a. The YBCO film was $330 \mathrm{~nm}$ thick in this case, the temperature $10 \mathrm{~K}$, and the applied field $17.3 \mathrm{mT}$. From this plot it is obvious that the velocity is much larger in the beginning (which we will call regime I) than later on (regime II). Qualitatively this can be ascribed to the fact that the driving force for the dendrite is expected to be largest in the region of high field gradients, i.e. close to the sample edge. About $10 \mathrm{~ns}$ after nucleation, the slope and hence the velocity becomes more or less constant at a value of $(18 \pm 2) \cdot 10^{3} \mathrm{~m} / \mathrm{s}$ (regime II), i.e. the dendrites are about $0.6 \mathrm{~mm}$ long when regime I changes to regime II. The velocity $v(t)$ derived from the slope of Fig. $4 \mathrm{a}$ is plotted in Fig. $4 \mathrm{~b}$, together with data points obtained by the double exposure technique mentioned above. Apparently the two methods lead to comparable results, although the scatter of the data is relatively large. The error bars in the $\mathrm{x}$-direction in Fig. $4 \mathrm{~b}$ give the delay elapsed between the two exposures.

The stage where the avalanche comes to a stop, is not easily accessible in a systematic way by the snapshot technique. However, we can conclude from the available data that the velocity does not display a gradual decrease, but drops to zero rather rapidly.

\section{Conclusions}

These investigations show that the dynamics of the garnet film is sufficiently fast to study processes in the sub-nanosecond regime like the dendritic flux 
instability. The magnetic instability in thin YBCO films display some prominent features: one can distinguish between two regimes of flux propagation, an early stage, where the dendrite velocity is high and decreasing in time, and a late stage, where the velocity is nearly constant. Further systematic studies like the influence of magnetic field and temperature can be found in [12].

\section{Acknowledgments}

We want to thank E. Il' yashenko for providing the garnet layers and $\mathrm{H}$. Kinder and $\mathrm{K}$. Numssen for providing the YBCO films. The work was supported by Optik Zentrum Konstanz.

\section{References}

[1] Wertheimer M. R., Gilchrist J. le G.; J. Phys. Chem. Solids 28 (1967) 2509

[2] Brüll P., Kirchgässner D., Leiderer P., Berberich P., Kinder H.; Ann. Physik 1 (1992) 243

[3] Herminghaus S., Boneberg J., Brüll P., Bujok V., Leiderer P.; Superconductivity and Particle Detection World Scientific, Singapore (1994) 85

[4] Durán C. A., Gammel P. L., Miller R. E. Bishop D. J. Phys. Rev. B 52 (1995) 75

[5] Bujok V., Brüll P., Boneberg J., Herminghaus S., Leiderer P. Appl. Phys. Lett. 63 (1993) 412

[6] Leiderer P., Boneberg J., Brüll P., Bujok V., Herminghaus S. Phys. Rev. Lett. 71 (1993) 2646

[7] Johansen T. H., Baziljevich M., Shantsev D. V., Goa P. E., Galperin Y. M., Kan W. N., Kim H. J., Choi E. M., Kim M.-S., Lee S. I. Europhys. Lett. 59 (2002) 599

[8] Bolz U. Ph.D. Thesis, Konstanz (2002)

[9] Dorosinskii L. A.,Indenbom M. V., Nikitenko V. I., Ossip'yan Y. A., Polyanskii, A. A. Vlasko-Vlasov V. K. Physica C 203 (1992) 149

[10] Brüll P., Kirchgässner D., Leiderer P. Physica C 182 (1991) 339

[11] Brandt E. H., Indenbom M., Forkl A. Europhys. Lett. 22 (1993) 735

[12] Bolz U., Schmidt D., Biehler B., Runge B.-U., and Leiderer P., Europhys. Lett. (2003) in print 